\title{
Pareto Frontier for Simultaneous Structural and Manufacturing Optimization of a Composite Part
}

\author{
Ghiasi $\mathrm{H}^{*+}$, Pasini $\mathrm{D}^{*}$, Lessard $\mathrm{L}^{*}$
}

\begin{abstract}
Optimized design of composite structures requires simultaneous optimization of structural performance and manufacturing process. Such a challenge calls for a multi-objective optimization. Here, a generating multiobjective optimization method called Normalized Normal Constraint Method [NNCM], which attains a set of optimal solutions and allows the designer to explore design alternatives before making the final decision, is coupled with a local-global search called Constrained Globalized Bounded Nelder-Mead [CGBNM] method. The proposed approach is applied to the design of a Z-shaped composite bracket for optimization of structural and manufacturing objectives. Comparison of the results with Non-dominated Sorting Genetic Algorithm [NSGA-II] shows that when only a small number of function evaluations are possible and a few Pareto optima are desired, the proposed method outperforms NSGA-II in terms of convergence to the true Pareto frontier. The results are validated by an enumeration search and by exploring the neighbourhood of the final solutions.
\end{abstract}

Keywords: Multi-objective Optimization, Composite materials, Pareto Frontier, Global Optimization, Simultaneous Optimization.

$n:$ Number of design variables

\section{Nomenclature}

$m:$ Number of objectives

$\mathfrak{R}^{n}$ : Design space of a problem with $n$ design variables

$S:$ Feasible region of the design space $\left(S \subset \Re^{n}\right)$

$Z$ : Criterion space $\left(Z=\vec{f}(S) \subset \mathfrak{R}^{m}\right)$

$\vec{x}^{*}$ : A Pareto optimum solution in the design space

$X^{*}:$ All Pareto optimal points in the design space,

$\vec{f}^{*}=\vec{f}\left(x^{*}\right)$ : A Pareto optimum in criterion space

$\bar{f}:$ Normalized objective

$\phi(x)$ : Probability of sampling a point $x \in S$

$r$ : Number of non-dominated solutions obtained during the optimization process;

\section{Introduction}

Because of their excellent mechanical properties, laminated fibrous composite materials are successfully used in a wide range of structural applications. However, due to the large number of design variables and objectives, the design of composite materials is more complex than the design of uniform isotropic materials. This is not only due to the anisotropic material properties but also because of the strong interconnection between design and manufacturing issues. Thus, a common way to simplify the composite

\footnotetext{
* McGill University, Department of Mechanical Engineering, Macdonald Eng. Bldg, 817 Sherbrooke St. West, Montreal, QC, Canada, H3A 2K6.

+ Corresponding author, E-Mail: hossein.ghiasi@mail.mcgill.ca, Phone:+1(514)398-6292 Fax: +1(514)398-7365
} 
design problem is to separate structural design from manufacturing design [Le Riche et al. 2003] and performing the latter just after the former is completed. However, researches by Wang and Costin (1992), Costin (1993), Henderson et al. (1999), Wang et al. (2002), Le Riche et al. (2003), and a series of papers by Park et al. $(2001,2003,2004,2005)$ have shown that a better overall performance is obtainable if structural and manufacturing objectives are simultaneously optimized. This task requires solving a Multi-Objective Optimization [MOO] problem, which is generally more challenging than solving a single-objective optimization problem and involves more computational effort.

This paper proposes using a method called Normalized Normal Constraint Method [NNCM] to simultaneously optimize several objectives in a composite design problem. To obtain each of the numerous possible solutions to this problem, NNCM performs a single-objective optimization. For the singleobjective optimization, a local-global search called Constrained Globalized Nelder-Mead [CGBNM] is suggested. The proposed method is applied to the design of a Z-shaped composite bracket, the goal of which is to find the fiber orientations and the geometrical parameters that maximize the strength and minimize the weight and the spring-in after demoulding. The results are compared to the ones obtained by one of the most successful multi-objective evolutionary optimization methods in the literature, called Nondominated Sorting Genetic Algorithm NSGA-II [Deb et al. 2002].

In the following sections, the main terminology and definitions used in MOO are provided before reviewing the applications of these methods in design of composite materials. The proposed MOO method, its internal single-objective method, and the two parameters used to measure the performance of a MOO method are explained in the third section. The fourth section applies the proposed method to the design of a Z-shaped composite bracket and compares the results with NSGA-II. Validation of the numerical results, discussion, and concluding remarks are given at the end.

\section{MOO and Design of Composite Materials}

In many applications, such as design with composite materials, usually there is more than one objective involved; this type of optimization problems is generally called Multi-Objective Optimization [MOO] problems. This section introduces the terminology used in MOO and reviews its applications in design of composite materials.

\subsection{Multi-Objective Optimization}

The process of systematically and simultaneously optimizing several objectives is called Multi-Objective Optimization [MOO]. A MOO problem is mathematically expressed as:

minimize $\vec{f}(x)=\left\{f_{i}(x): S \mapsto \Re ; i=1, \ldots, m ; m>1\right\}$

subject to $\left\{\begin{array}{l}g_{j}(x) \geq 0 ;\left\{g_{j}(x): S \mapsto \Re ; j=1, \ldots, J ; J \geq 0\right\} \\ h_{k}(x)=0 ;\left\{h_{k}(x): S \mapsto \Re ; k=1, \ldots, K ; K \geq 0\right\}\end{array}\right.$

The feasible region, $S$, in (1) is defined as:

$S=\left\{x \in R \mid g_{j}(x) \geq 0 \& h_{k}(x)=0 ; j=1, \ldots, J ; k=1, \ldots, K\right\}$

The objectives in this problem are in contrast to each other, and there is no unique solution to a problem with more than one conflicting objective. There exist a number of solutions which all are optimum. These solutions are called Pareto optimum solutions [Pareto 1906]. The definition of Pareto optimality is based on domination that follows.

Definition 1 (Domination): Considering the optimization problem formulation in (1), a solution $x_{1} \in S$ dominates a solution $x_{2} \in S$, if $x_{1}$ is smaller than $x_{2}$ in at least one objective and is not bigger with respect to the other objectives.

$x_{1}$ dominate $x_{2} \Leftrightarrow\left\{\begin{array}{l}\forall i: 1 \leq i \leq m \Rightarrow f_{i}\left(x_{1}\right) \leq f_{i}\left(x_{2}\right) \\ \exists j: 1 \leq j \leq m: f_{j}\left(x_{1}\right)<f_{j}\left(x_{2}\right)\end{array} \wedge\right.$ 
Definition 2 (Pareto Optimal): Considering the problem formulation (1), a solution $x^{*} \in S$ is Pareto optimal if and only if it is not dominated by any other solution in $S$. Collection of all Pareto solutions is called Pareto frontier, represented by $X^{*}$.

$x^{*} \in X^{*} \Leftrightarrow \nexists x \in S: x$ dominate $x^{*}$

There are numerous methods for multi-objective optimization, and they can be classified in many ways according to different criteria. A comprehensive review of these methods can be found in Miettinen (1999), Deb (2001), and Marler et al. (2004). For the purpose of this paper, we categorize these methods into two distinct groups:

1) Non-generating methods that find only one Pareto solution for a given MOO problem. The Pareto solution may be selected with or without considering the user's preferences. If the user preferences are required, they may be obtained at the onset of the optimization process (method with priori preferences) or interactively as the optimization process goes on (methods with progressive preferences).

2) Generating methods that generate a set of Pareto optimal solutions among which the user chooses the final solution according to his/her preferences. These methods are also called methods with posteriori preferences.

This section reviews the application of different $\mathrm{MOO}$ methods to the design of composite materials, which is the focus of this paper.

\subsection{Multi-Objective Design of Composite Materials}

Current literature on MOO of composite materials reveals mainly the use of non-generating methods. Although these are preferred for their simplicity and for being less time consuming; there are attempts of using generating methods to design a composite part. Both methods are reviewed in this section.

Method of weighted-sum is the most common MOO method used in composite design. In this nongenerating method, the objectives are combined into a single-objective problem using user-defined weighting factors. The resulted single-objective optimization problem may be solved by any optimization technique. Examples of application of this method in stacking sequence design of composite materials can be found in Adali (1983), Walker et al. (1997), Manne and Tsai (1998), Walker and Smith (2003), Deka et al. (2005), Jyotideka et al. (2005), and Mohan Rao and Arvind (2005), and Abouhamze and Shakeri (2007). Kere et al. $(2002,2003)$ also used the weighted-sum, but only to reduce their multi-objective optimization problem to a bi-criterion problem. The most sensitive objective was used as the first criterion, while the others were collected in a weighted sum as the second criterion. The resulted bi-criterion problem was solved by using a layerwise approach, in which all possible permutations of adding a new layer to the laminate were examined, and the one that made the maximum improvement in either of the two criteria was accepted.

Another strategy, also classified among non-generating methods, is to optimize one criterion while constraining the others to user-defined limits. These methods are generally known as " $\varepsilon$-constraint methods" and are commonly used in composite design optimization. Wang and Costin $(1992,1993)$ found the minimum weight design of a composite shell by applying constraints on manufacturing objectives. Henderson et al (1999) integrated manufacturing considerations as constraints into the design optimization of a blade stiffened panel. Park et al. (2001) applied constraints on processing time and panel stiffness to minimize the weight of a plate made by RTM. Wang et al. (2002) optimized the number of ribs and spars in an aerospace composite structure using weight as the primary design drive and the cost parameter as a constraint. Le Riche et al. (2003) used the same method but frequently exchanged the objective and constraints.

Reference point method is another non-generating MOO method that has been used in composite design applications [Saravanos and Chamis 1992, Kere and Lento 2005]. This method minimizes an achievement function based on a reference point. The reference point, defined by the designer, is a feasible or infeasible point in the criterion space that is reasonable or desirable to the designer. The achievement function may be the Euclidian distance to the reference point or any other user-defined measure. Appropriate selection of the reference point has a major effect on the final solution obtained by this method. 
There are some non-generating methods with no user-defined preferences; an example is the min-max strategy, in which only the critical objective is optimized. For instance, if the goal of a problem is to maximize the strength of several components within an assembly, only the part with the minimum strength is considered in each step of the optimization process. The limitation is that this method requires the objectives to have comparable values. It is particularly useful for stress or stiffness minimization [Suresh et al. 2007].

Non-generating methods generally require an insight into the problem because the preference parameters (e.g. weighting factors, constraint values, reference point, etc.) must be set by the designer. These methods find only one optimal solution; however, they can also be used to generate a set of Pareto optimal solutions by varying the user-defined preference parameters [Watkins and Morris 1987, Adali et al. 1996, Mohan Rao et al. 2005], but the resulted solutions may not be uniformly spread along the Pareto frontier. In contrast, generating methods have the advantage of not requiring any user-defined preferences and generating a set of optimum solutions. The penalty, on the other hand, is that they usually need a great deal of computation. Non-dominated Sorting Genetic Algorithm [NSGA] and particle swarm are examples of methods falling in this group that are used for optimization of composite materials.

Non-dominated Sorting Genetic Algorithm [NSGA] was proposed by Srinivas et al. (1995) as an evolutionary method based on genetic algorithm [GA]. NSGA differs from simple GA only in the way the selection operator works. Before selection, individuals are ranked according to the level of non-domination. Each solution assigned a fitness equal to its non-domination level ( $1^{\text {st }}$ is the best level), thus minimization of the fitness promotes non-dominated solutions and eventually reaches the Pareto frontier. The original NSGA has no control on the spread of the obtained solutions. Deb et al. (2002) overcome this problem by adding a crowded control parameter to NSGA. The new method, called NSGA-II, is an elitist nondominated sorting GA that provides a set of non-dominated solutions uniformly distributed on Pareto frontier. Using mathematical test functions, Deb et al. (2002) reported that NSGA-II outperforms other contemporary multi-objective evolutionary methods in terms of convergence to the true Pareto frontier and achieving a uniform distribution of solutions. An Integer-coded version of NSGA-II was used by Pelletier and Vel (2006) to find Pareto-optimal designs for a composite laminates.

Particle Swarm Optimization [PSO] is another population-based, stochastic optimization method used in design of composite materials. Inspired by the flocking behaviour of the birds, each solution in this method is called a "particle" and resembles a bird among others. Each bird adjusts its position according to its own flying experience (best solution in its individual history) and the flying experience of the others (the best solution among all particles). There are several methodologies using PSO to handle problems with multiple objectives, among them Vector Evaluated PSO [VEPSO], a multi-swarm variant of PSO, was used by Omkar (2008) to minimize weight and cost of laminated composite components. VEPSO considers $m$ swarms, each consisting of $n$ particles. Each swarm is exclusively evaluated according to one of the objective functions. The adjustment process takes place according to the flying experience of the particle itself, and the particles in one of the other swarms. Although its performance is reported to be "satisfactory"; no comparison with other methods has been found by the authors.

A population-based generating MOO, such as NSGA-II or VEPSO, is computationally time consuming because it needs numerous function evaluations. Composite design problems have the particularity of having a time consuming function evaluation process, which usually involves performing several finite element analysis. Therefore, in many cases, it may not be possible or desirable to perform as many function evaluations as is required for a population-based method to converge. The other property of populationbased methods is that they usually return a set of non-dominated solutions almost as large as their population size. To facilitate the selection of the final solution, a designer may prefer to have only a few solutions with a low computational cost rather than a large number of alternative solutions with a high computational cost. For these situations, this paper proposes a combination of a local single-objective optimization technique and a MOO approach called NNCM. The next section explains the proposed method, the single-objective optimization method and how the latter is embedded in the former. 


\section{Optimization Procedure}

Among generating methods, a survey of which can be found in Marler (2004), Miettinen (1999), and Deb (2001), Normalized Normal Constraint Method [NNCM] [Messac et al. 2003] is implemented in this work. This method normalizes the design space and introduces new constraints. Considering the new constraints, optimization of only one of the objectives returns a non-dominated solution. When several of these singleobjective optimization problems are solved, several non-dominated solutions are obtained. The difference between this method and varying user preferences in a non-generating method is that here the set of constraints are introduced to spread the final solutions uniformly in the criterion space. This section explains the NNCM method, the single-objective optimization method and their integration. Finally, two parameters are presented to measure the performance of a MOO method.

\subsection{Normalized Normal Constraint Method (NNCM)}

Normalized Normal Constraint Method [NNCM] is an algorithm for generating a set of evenly spaced solutions on a Pareto frontier [Messac et al. 2003]. This method yields Pareto optimal solutions, and its performance is independent of the scale of the objective functions. NNCM method and some related definitions are presented in this section.

Definition 3 (Utopia Point): Considering optimization problem (1), a point $\vec{f}^{o} \in Z$ in the criterion space is called a utopia point if and only if:

$f_{i}^{o}=\min _{x}\left\{f_{i}(x) \mid x \in S\right\} ; i=1, \ldots, m$.

Because of contradicting objectives, the utopia point is unattainable.

Definition 4 (Anchor Point): A non-dominated point $\vec{f}^{* *} \in Z$ is an anchor point if and only if it is Pareto optimal and at least for one $i=1,2, \ldots, m ; f_{i}^{* *}=\min _{x}\left\{f_{i}(x) \mid x \in S\right\}$.

The first step in NNCM is to normalize the design space. For this purpose, the utopia and the anchor points are required. These points are found by optimizing only one of the objectives at a time. After finding these points, the criterion space is normalized using the following transformation.

$\bar{f}_{i}=\frac{f_{i}-f_{i}^{o}}{f_{i}^{\max }-f_{i}^{o}}$

$f_{i}^{\max }=\max \left\{f_{i}(x) ; x \notin X^{*}\right\}$

The normalization process locates the utopia point at the origin and the anchor points at the unit coordinates. Figure 1-a shows the original criterion space and the Pareto frontier of a generic bi-criterion problem. Figure 1-b represents the Pareto frontier of the same problem after normalization.

The next step is to form the utopia hyperplane, which is a hyperplane with vertices located at the anchor points. For a bi-criterion problem, the utopia hyperplane is a line as shown in Figure 1-c. Next, a grid of evenly distributed points on the utopia hyperplane is generated. The number of points in this grid is defined by the user. Figure 1-c shows, for example, a grid of six points on the utopia line. If these points are projected onto the Pareto frontier, several Pareto optimum solutions are obtained. To find the Pareto optimum solution corresponding to each point in this grid, a single-objective optimization problem must be solved. This problem entails minimizing one of the normalized objectives with an additional inequality constraint. For example, the Pareto optimum solution corresponding to point " $P$ " in Figure 1-c can be found by minimizing $\bar{f}_{2}$ while the feasible region is cut by the line passing through this point and perpendicular to the utopia line. The feasible region of this single objective optimization problem is shown in Figure 1-c. The solution of this problem, $\bar{f}^{*}$, is a Pareto optimum solution for the original multiobjective problem. Other Pareto optimal points can be found by repeating the same procedure for other points on the utopia line.

If the objective functions have many local optima or the Pareto frontier is discontinuous, it is possible to have some dominated solutions among the final solutions. Composite stacking sequence design problem is 
well known for having many local optima; therefore, dominated solutions are expected. Messac et al. (2003) proposed a filter that removes all dominated solutions, after all the single-objective

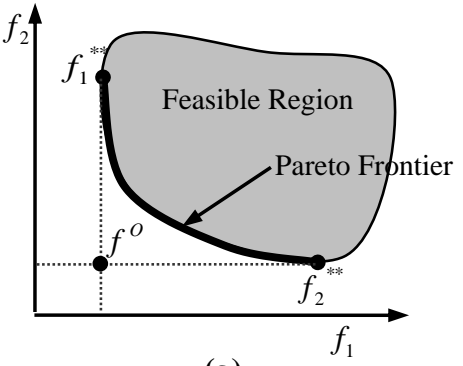

(a)

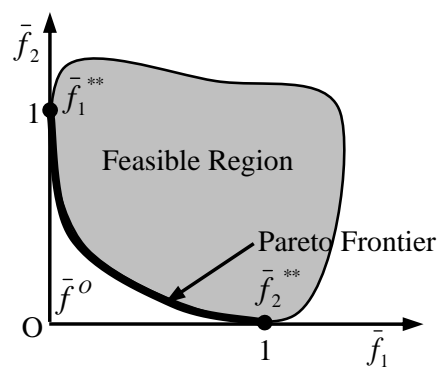

(b)

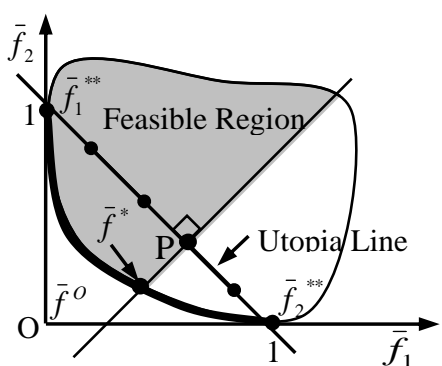

(c)

Figure 1. (a) A typical bi-criterion space (b) normalized criterion space (c) a normal constraint introduced by NNCM and the feasible region of the resulted single-objective problem $\left(\min \bar{f}_{2}\right)$

optimizations are completed. This filter requires a pair wise comparison of all the solutions. Since this algorithm aims at finding a small number of solutions on the Pareto frontier (e.g. around ten points) the performance of the filtering algorithm is of minor concern and not discussed here.

\subsection{CGBNM for Single-Objective Optimization}

In order to find each Pareto optimum solution, NNCM requires solving a single-objective optimization problem. Since this algorithm is proposed for solving a composite design problem, in which the gradients of the objectives are not available, a direct optimization method is required. On the other hand, considering the time consuming process of structural and manufacturing analysis of a composite part, an evolutionary algorithm may not be a good choice due to the low rate of convergence. Therefore, a local-global search called CGBNM [Ghiasi et al. 2008] is used for this purpose. CGBNM, which stands for Constrained Globalized Bounded Nelder-Mead, is an improved version of the algorithm introduced by Luersen et al. (2004). This method is using several restarts of a simplex optimization method called Nelder-Mead [N-M] method (Nelder and Mead 1965). The restart procedure uses a probability distribution function that initiates $\mathrm{N}-\mathrm{M}$ local search from new points far from previously sampled ones. CGBNCM is capable of finding several local optima for a multi-modal function. It has been shown that CGBNM is more efficient than an evolutionary algorithm, when a small number of function evaluations are possible [Ghiasi et al. 2008].

The flowchart of CGBNM is shown in Figure 2. The main blocks marked by the gray pattern and the bold border, are: "Nelder-Mead local optimizer" and "Restart procedure". The first finds a local optimum, while the second restarts the local search to confirm its convergence to a true optimum or to help finding another local solution. The maximum number of iterations for each restarts and the total number of function evaluations are defined by the user at the onset of the process.

Nelder-Mead [N-M] sequential optimization method, proposed by Nelder and Mead (1965), is among the most popular direct methods for local optimization of unconstrained problems. N-M method compares the objective values at a set of $n+1$ points called a simplex. The simplex is moved toward the optimum solution by four operators: reflection, expansion, contraction, or shrinkage. Reflection operator mirrors the worst point in the simplex with respect to the other points in that simplex. This is the basic step that moves the simplex toward a better solution. If the new point is better than the old point, the move is expanded by using the expansion operator, else it is contracted by using the contraction operator. If neither of these operators could find a better point, the simplex is shrunk toward its best point. The process is terminated when the simplex converges to an optimum. It has been shown that this method is effective in practice by producing a rapid initial decrease in function values [Lagarias et al. 1998], but the local optimum found is dependent on the initial simplex [Humphrey and Wilson 2000]. Therefore, in CGBNM a probability function is used to restart the local search from new points far from previously inspected regions in the design space. 
The restart procedure re-initializes N-M in order to ensure that the solution found in the last try is a local optimum (small test or large test) or to find a new local optimum (probabilistic restart). The small test and

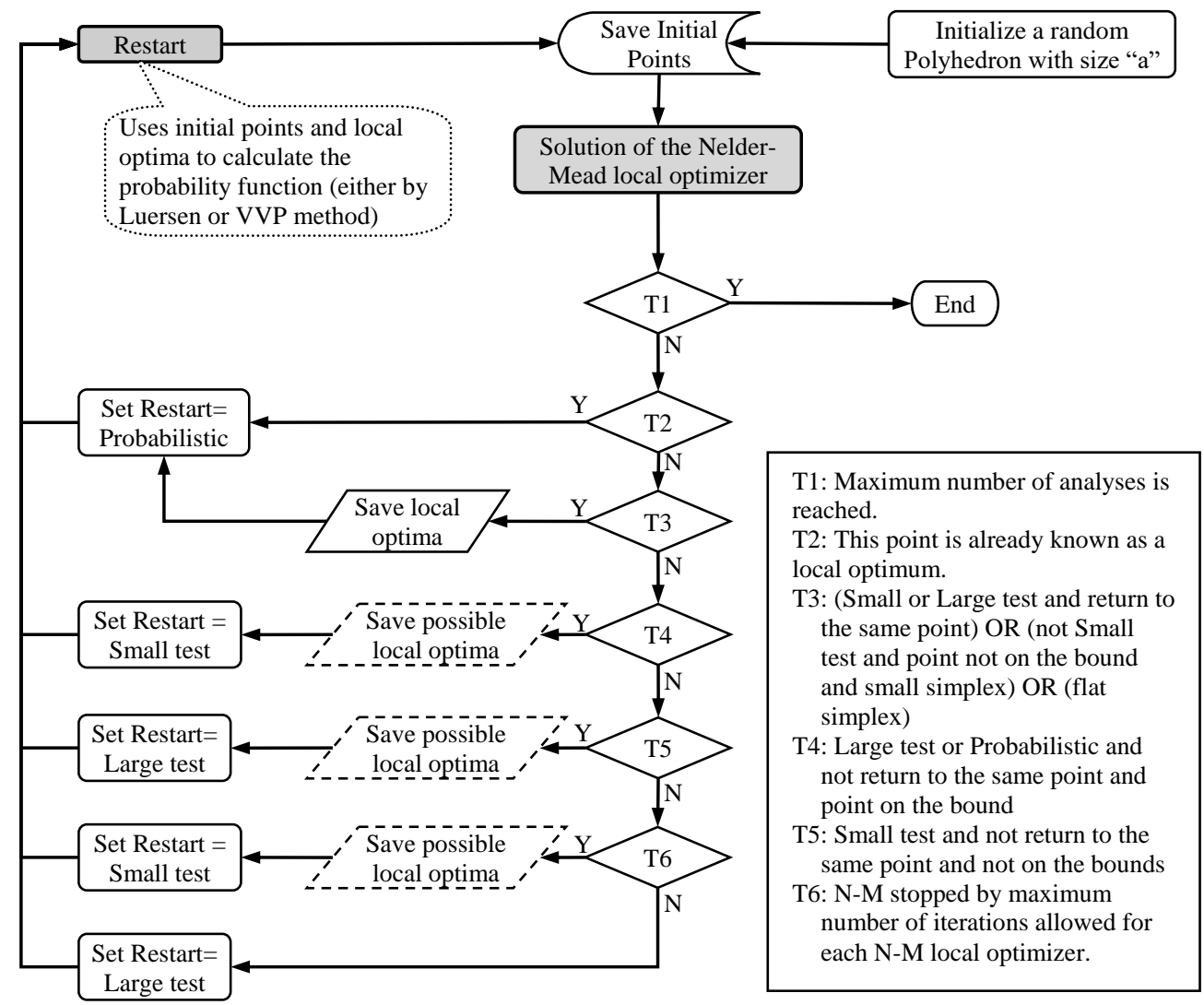

Figure 2. Restart and convergence tests linking in CGBNM

large test restart the N-M from the last solution obtained with a simplex of size $a_{s}$ or $a_{l}$, user-defined parameters. If the new small or large simplex returns to the same solution, the result is saved as a local optimum, and the N-M is initialized with a probabilistic restart. The probabilistic restart initiates the local search from a simplex located in the region that has not been previously explored. This strategy eases the finding of a new local optimum. The probabilistic restart procedure uses a one-dimensional adaptive probability function called Variable Variance Probability [VVP]. Using VVP, probability of sampling point $x$ is calculated as follow:

$\phi(x)=\frac{1}{\sqrt{2 \pi} \sigma}\left(1-e^{-\left(d_{\min }^{2} / 2 \sigma^{2}\right)}\right)$

$d_{\text {min }}=\min _{i=1, \ldots, I}\left\{d_{i}=\sqrt{\sum_{k=1}^{n}\left(\frac{x_{k, i}-x_{k}}{x_{k u}-x_{k l}}\right)^{2}}\right\}$

$x_{i} \mathrm{~s}$ in this equation are the points in the design space that are previously sampled. $d_{\min }$ is the minimum non-dimensional Euclidian distance between point $x$ and one of these points. The variance of the normal probability density, $\sigma$, is updated in each restart by using the following equation:

$\sigma=\frac{1}{3 \sqrt[n]{m}}$

Then a selection pool is created, in which each point has a number of copies proportional to its probability value. Therefore, points far from previously sampled points have more chance to be selected as an initial point for the next local optimization. 
N-M method in its original form is unable to deal with nonlinear constraints; however, a composite design problem is often constrained by several nonlinear constraints, such as failure criteria, and others that may emerge by using NNCM. Therefore, it is important that the selected optimization method be able to handle these constraints. In CGBNM, this goal is achieved by using a repair procedure that brings the infeasible solutions into the feasible region. This procedure consists of a backtracking scheme; when a new point generated by reflection or expansion violates one of the nonlinear constraints, the new point is moved toward the original feasible point such that the distance between these two points is reduced by a factor $\alpha \in(0,1)$. The procedure is terminated when either a feasible point is found or a predetermined number of trials is reached. If the procedure fails to find a new feasible point, the original point is kept, and the simplex is shrunk towards its best vertex.

\subsection{Performance Measures}

Two performance metrics used by Deb et al. (2002) to measure the performance of a non-generating MOO method are presented in this section. These two parameters are used later to compare the proposed method with NSGA-II. The first metric, $\gamma$, measures the extent of convergence to a known set of Pareto-optimal solutions, while the second, $\Delta$, measures the extent of spread achieved among obtained solutions. Both metrics are positive numbers and are desired to be as small as possible.

Convergence of a set of solutions to a known Pareto frontier is measured by the average of the minimum distance of all the solutions from the Pareto frontier. To find the minimum distance from the Pareto frontier, a grid of uniformly distributed points on Pareto frontier are generated. The minimum distance of a solution from one of the points in this grid is used as the minimum distance to Pareto frontier. The convergence metric, $\gamma$, is mathematically defined as below:

$\gamma=\frac{1}{p} \sum_{i=1}^{p} d_{i} ; d_{i}=\min _{j}\left\|\vec{f}_{i}-\vec{f}_{j}^{*}\right\|$

In this equation, \|\| shows the Euclidian distance between the two points in the criterion space. Figure 3-a illustrates how this metric is calculated for a bi-criterion problem.
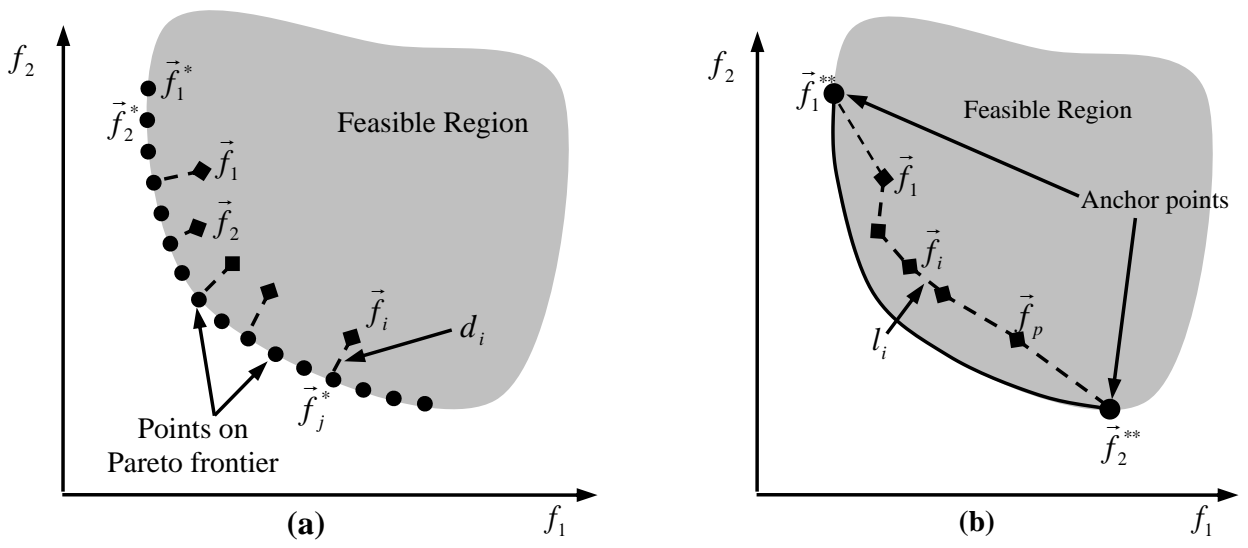

Figure 3. (a) Convergence metric $\gamma$, (b) diversity metric, $\Delta$ [Deb et al. 2002]

The second metric, $\Delta$, provides information about the extent of spread achieved among the obtained solutions. It is desired that a set of solutions obtained by a generating MOO spans the entire Pareto-optimal region and is uniformly distributed along the Pareto frontier. The following equation is used to calculate this metric for a bi-criterion problem:

$\Delta=\frac{l_{0}+l_{p}+\sum_{i=1}^{p-1}\left(l_{i}-\bar{l}\right)}{l_{0}+l_{p}+(p-1) \bar{l}}$

$\bar{l}=\frac{1}{p-1} \sum_{i=1}^{p-1} l_{i}$ 
As shown in Figure 3-b, $l_{0}$ and $l_{p}$ of the above equation are the Euclidian distances between the extreme solutions and the anchor points. $l_{i}$ is the Euclidian distance between two solutions. This metric is zero if the solutions are equally spaced and includes both anchor points.

\section{Composite Test Case and Numerical Results}

In this section, NNCM is applied to the simultaneous structural and manufacturing optimization of a Zshaped composite bracket shown in Figure 4. The bracket is made of 16-ply balanced symmetric laminate of graphite/epoxy (AS4/8552) with fiber orientation $\left[ \pm \theta_{1} / \pm \theta_{2} / \pm \theta_{3} / \pm \theta_{4}\right]_{s}$. The goal is to find geometries and lamination sequences that minimize weight and spring-in and maximize strength. The part should not fail or delaminate anywhere and should satisfy a safety factor of 1.5 against failure and 2 against delamination. Delamination is calculated in the curved regions where the angle shape causes high interlaminar normal stresses. The vertical deflection of less than $1 \mathrm{~mm}$ and the spring-in of less than $0.5^{\circ}$ are strictly required for an acceptable design. The semi-analytical models of first-ply-failure, delamination, deflection, and spring-in used for the optimization process are described in Appendix 1.

\subsection{Optimization Set up}

The MOO method described in section three is used to solve this composite design problem. The objective functions are divided into two groups of manufacturing objectives and structural objectives. Considering these two group of objectives, NNCM is used to find the Pareto solutions.

The first group of objectives deals with the structural performance including the weight minimization and strength maximization. The two objectives are grouped into one weighted-sum as:

$\min f_{s}=\frac{W(g r)}{5 g r}-\frac{R}{1.5}$

where $W$ is the weight, and $R$ is the load factor. The second group of objectives deals with the manufacturing aspect and includes only minimization of spring-in after demoulding.

$\min f_{m}=|\Delta \theta|$

By incorporating the set of inequality constraints described in the problem definition, the optimization problem is formulated as follow:

$\min \left\{f_{s}(x), f_{m}(x)\right\} ; x=\left\{\theta_{1}, \theta_{2}, \theta_{3}, \theta_{4}, e, s_{r}, r\right\}$

Subject to $\left\{\begin{array}{l}\left\{R(x) \geq 1.5 \wedge D(x) \geq 2.0 \wedge S_{r}(x) \geq 10 \mathrm{~mm} \wedge|\delta(x)| \leq 1 \mathrm{~mm} \wedge|\Delta \theta(x)| \leq 0.5^{\circ}\right\} \\ \theta_{i} \in\left[-90^{\circ}, 90^{\circ}\right] ; i=1, \ldots, 4 ; \\ e \in[0,0.15](\mathrm{m}) ; \\ s_{r} \in[2,5](\mathrm{cm}) ; \\ r \in[2,20](\mathrm{mm}) ;\end{array}\right.$

Where $D(x)$ in this equation is the delamination factor. $S_{r}, e$ and $r$ are the geometrical parameters shown in Figure 4. $\delta(x)$ is the maximum vertical deflection, and finally $\Delta \theta(x)$ is the spring-in.

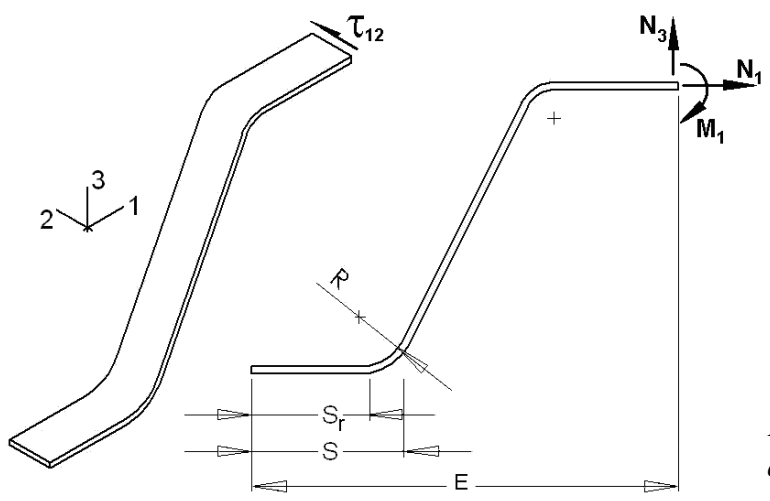

$$
\begin{aligned}
& \mathrm{N}_{1}=500 \mathrm{~N} / \mathrm{m} \\
& \mathrm{N}_{3}=100 \mathrm{~N} / \mathrm{m} \\
& \mathrm{M}_{1}=50 \mathrm{~N} \cdot \mathrm{m} / \mathrm{m} \\
& \tau_{12}=300 \mathrm{~N} / \mathrm{m}
\end{aligned}
$$

Figure 4. Geometrical variables and applied loads on the composite bracket 
The first step of NNCM is to find the utopia and the anchor points. To this end, two single-objective optimization problems must be solved; one optimizes only the structural objectives while the manufacturing objective is set free to take any value. The second problem minimizes only the manufacturing objective and the structural ones are ignored. We refer to the solution of the first problem as structural-only solution $\left(f_{s}^{* * *}\right)$, and the solution of the second problem as manufacturing-only solution $\left(f_{m}^{* *}\right)$.

$$
f_{s}^{* * *}=(0,|-0.149|)=(0,0.149) ; f_{m}^{* *}=\left(\frac{9.47 g r}{5 g r}-\frac{1.51}{1.5}, 0\right)=(0.886,0) ;
$$

The utopia point does not correspond to any physical design and is calculated as follow.

$\vec{f}^{o}=\left(f_{s}^{o}, f_{m}^{o}\right) ; f_{s}^{o}=\frac{6.99 g r}{5 g r}-\frac{2.15}{1.5}=-0.037 ; \quad f_{m}^{o}=|-0.0048|=0.0048$.

Figure 5 shows the two anchor points, $f_{s}^{* *}$ and $f_{m}^{* *}$, in the normalized criterion space. The corresponding lamination sequences and 2D cross-section shapes are also represented beside each point. Crossed-line diagrams in this figure and Figure 6 represent the fiber orientations in the bracket. Vertical lines represent the fibers running along the length of the bracket, whereas horizontal ones show the fibers running along the width of the bracket (normal to the cross-section).

Regarding the objective values at Utopia and anchor points the normalization is performed using the following transformation.

$\bar{f}_{s}=\frac{f_{s}(x)+0.037}{0.923} ; \quad \bar{f}_{m}=\frac{f_{m}(x)-0.005}{0.144}$

\subsection{Numerical Results}

NNCM requires solving a set of single-objective minimization problems with an additional nonlinear constraint. The additional nonlinear constraint makes the feasible region tighter, thus the solution to the single-objective minimization problem would be different than the anchor points. Here, two different scenarios are considered. In the first scenario, called minStr, the structural objective is minimized. In contrast, the second scenario, called $\operatorname{minM} f g$, minimizes the manufacturing objective. Each scenario uses three values for the constraints, seeking three points on the Pareto frontier (i.e. A, B, and C in Figure 5).

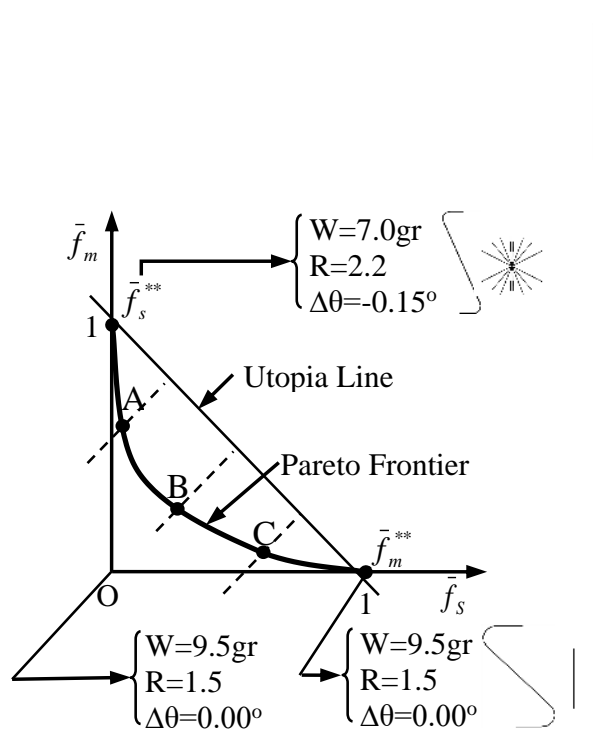

Figure 5. Utopia point, two anchor points, utopia line and the user-selected points for composite bracket design problem

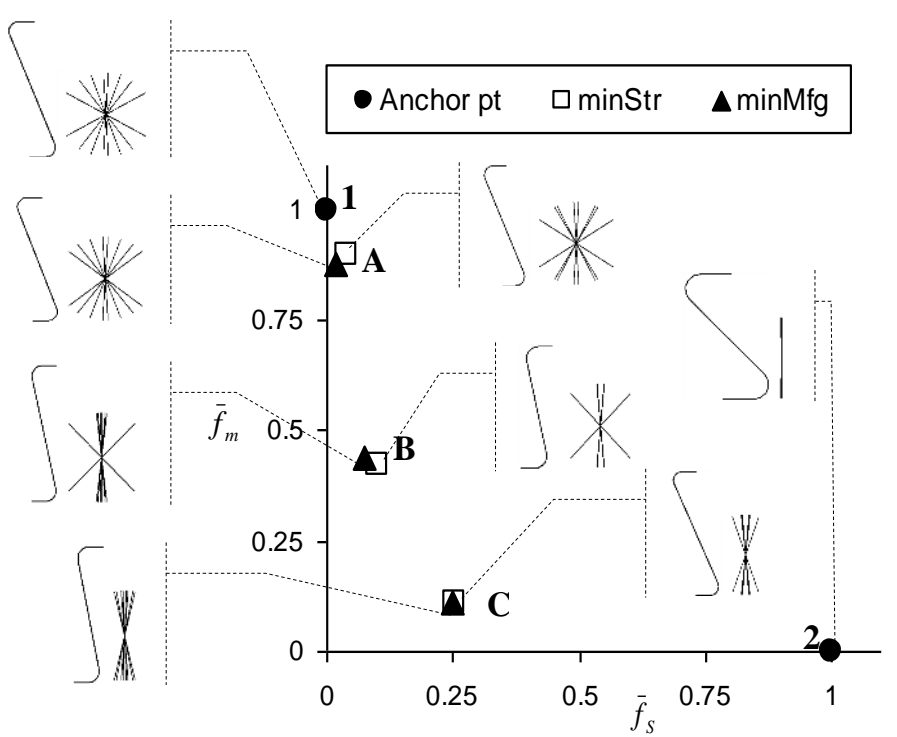

Figure 6. Graphical representation of $2 D$ cross-section and lamination sequences of the solutions found on the Pareto frontier. Crossed-line diagrams represent the fiber orientations. 
Table 1. The performance criteria at the two anchor points and at three other points on the Pareto frontier

\begin{tabular}{cccccc}
\hline Point & $\mathbf{1}$ & $\mathbf{A}$ & $\mathbf{B}$ & $\mathbf{C}$ & $\mathbf{2}$ \\
\hline Weight (gr) & 7.0 & 7.0 & 6.9 & 7.0 & 9.5 \\
Deflection (mm) & 0.046 & 0.073 & 0.099 & 0.166 & 0.133 \\
Load Factor & 2.2 & 2.1 & 2.0 & 1.8 & 1.5 \\
Spring-in (degree) & -0.15 & -0.14 & -0.07 & -0.02 & -0.00 \\
\hline
\end{tabular}

The optimization problem in the first scenario, minStr, is formulated as:

$\left\{\begin{array}{l}\min \bar{f}_{s}(x) ; \quad x=\left\{\theta_{1}, \theta_{2}, \theta_{3}, \theta_{4}, e, s_{r}, r\right\} \\ \text { S.T.: }\left\{R(x) \geq 1.5 \wedge D(x) \geq 2.0 \wedge S_{r}(x) \geq 10 m m \wedge|\delta(x)| \leq 1 m m \wedge|\Delta \theta(x)| \leq 0.5^{\circ} \wedge \bar{f}_{s}-\bar{f}_{m}+a \geq 0\right\}\end{array}\right.$

The last constraint in (14) is added by NNCM, in which the constant $a$ in is set to $-0.5,0$, and 0.5 to obtain points $\mathrm{A}, \mathrm{B}$, and $\mathrm{C}$ in Figure 5. The optimization problem for the second scenario, minMfg, is identical to the first one but involving minimization of $\bar{f}_{m}$ and replacing the last constraint with $\bar{f}_{m}-\bar{f}_{s}+a \geq 0$.

Figure 6 shows the results obtained in two scenarios, minStr and minMfg, each including 2000 function evaluations. As expected, the points obtained in these two scenarios are similar regarding the objective values. Points $\mathrm{A}, \mathrm{B}$ and $\mathrm{C}$ in this figure are shown by their normalized objective values. The optimized geometry (left) and lamination sequence (right) of the corresponding bracket are also shown for each data point.

Table 1 summarizes the objective values of the optimal solutions. As the design point moves from the structural-only design (point 1) toward manufacturing-only design (point 2), a) the geometry gradually changes from small brackets to elongated ones and $b$ ) the fiber orientations are changed from laminates in which fibers are distributed in all directions to laminates where fibers are mostly aligned at zero degrees.

The Pareto curve helps the designer better understand the trade-off between the conflicting objectives. For instance, the results have shown that starting from the best manufacturing design, penalizing only $10 \%$ the manufacturing objective (e.g. moving from a point with $\bar{f}_{m}=0$ to $\bar{f}_{m}=0.1$ ), the structural objective will improve more than $75 \%$ (e.g. is reduced from $\bar{f}_{s}=1$ to $\bar{f}_{s}=0.25$ ). However, this is not valid at the point with $\bar{f}_{s}=0.25$, where $10 \%$ penalty on manufacturing objectives results in almost equivalent improvement in structural performance.

\subsection{Validation and Interpretation of the Results}

In this section, the optimization procedure is validated by comparing the results to the ones obtained by a rough enumeration search over the design domain and a non-dominated sorting genetic algorithm (NSGA). The local optimality of the results is also demonstrated by analysing the neighbourhood of the optimal solutions.

This problem, similar to all other composite design problems, has many local optima and the global optima are unknown. Thus, to judge the validity of the results we resort to an enumeration search. Obtaining all possible fiber orientations and geometries requires numerous function evaluations (i.e. $10^{10}$ function evaluations for this problem). Here, we decide to examine all possible designs with fibers oriented at 0 , \pm 45 , and 90 degrees each with 64 different geometries, which still requires more than 5000 function evaluations.

Figure 7 shows the results of the enumeration search in the normalized criterion space beside the solutions found by NNCM. The solutions obtained by NNCM are located at the boundary of the feasible region. The unexplored region, right of these solutions, is resulted by the choice of having examined fiber orientations only at $0, \pm 45$, and 90 degrees. A valuable result is that the solutions found by NNCM dominate the optimum solutions found by the enumeration search, and it captures the overall shape of the boundary of the feasible region. For instance, at the lower-right corner of this figure, the feasible region is tangent to the horizontal axes. This property is also reflected by plotting a line passing through solutions found by NNCM. 


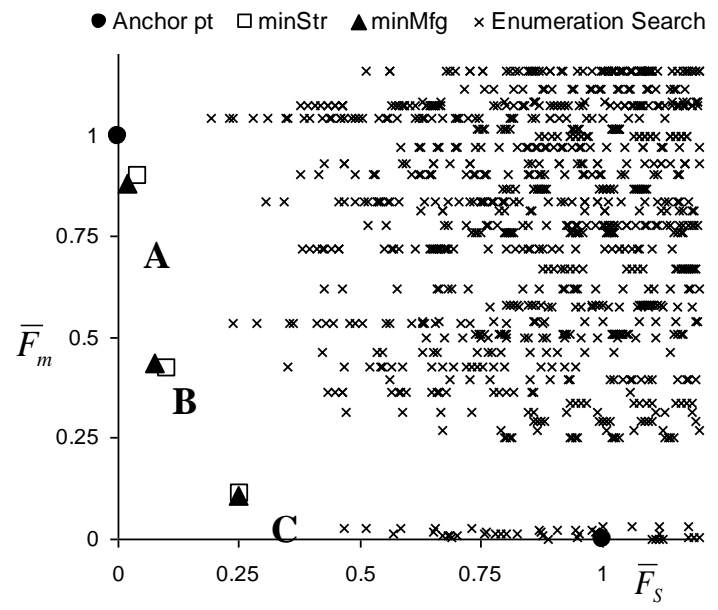

Figure 7. Enumeration search and the points on Pareto frontier that obtained by NNCM

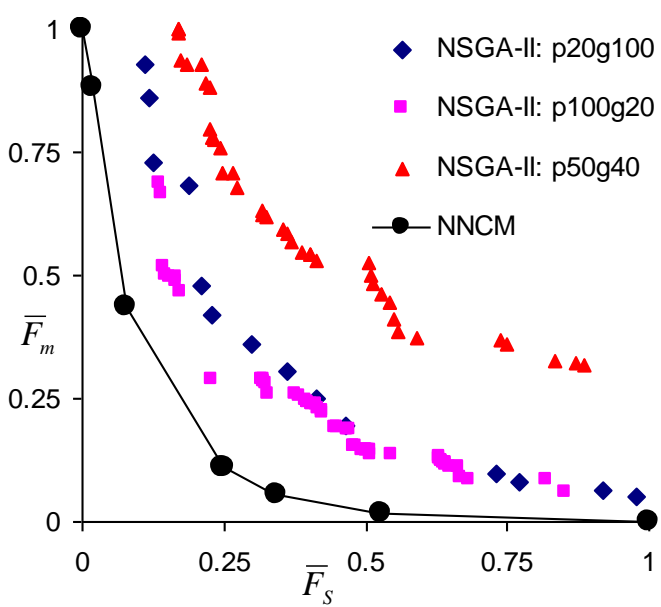

(a)

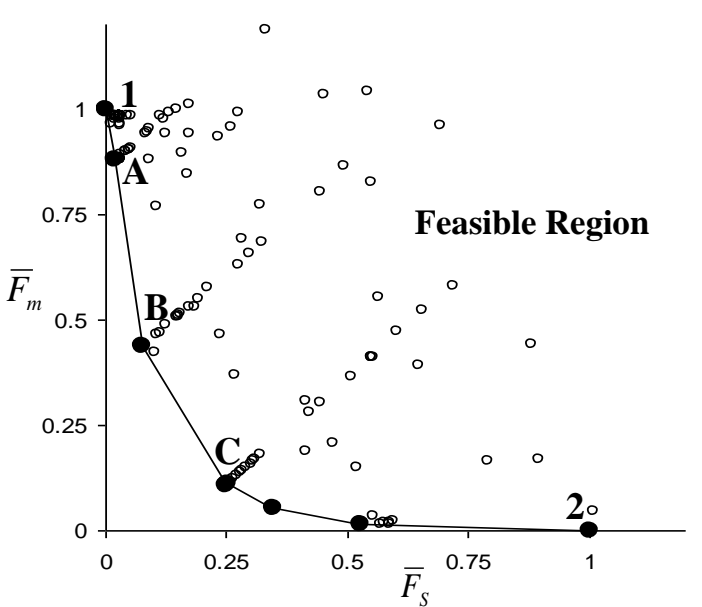

(b)

Figure 8. (a) Comparison of the Pareto frontier found by NNCM and NSGA (b) all local solutions obtained by NNCM coupled with GBNM

\subsubsection{Comparison with NSGA}

Evolutionary algorithms are commonly used in design of composite materials and also to solve MOO problems. Thus, here the result obtained by NNCM is compared to those obtained by NSGA-II. The comparison is made with respect to the performance parameters described in section 3.3. Both methods are applied to the composite design problem with maximum 2000 function evaluations.

Performance of NSGA-II is dependent on the population size and the number of generations. Three different cases were examined and the results are shown in Table 2. Each case is labelled with the population size and number of generations; for instance the case labelled as p50n40 has the population size of 50 chromosomes and the optimization process was continued till 40 generations. Number of generations and population sizes are selected such that, in each case, the total number of 2000 function evaluations is performed. The optimal solutions obtained are presented in Figure 8-a. This figure clearly shows that the solutions obtained by NNCM dominate all NSGA-II's solutions. Table 2 compares the same results in terms of the performance metrics introduced in section 3.3. Since the real Pareto frontier of the composite design problem is unknown, the origin and the two anchor points are used to calculate $\Delta$. This table shows 
that the NNCM can achieve a better convergence to the Pareto frontier than all NSGA-II runs with almost similar level of spread to the best results obtained by NSGA-II.

Table 2. Performance parameters, $\gamma$ and $\Delta$ and number of non-dominated solutions, $r^{\prime}$,

\begin{tabular}{rccc}
\multicolumn{4}{c}{ obtained by NNCM and NSGA-II } \\
\hline & $\gamma$ & $\Delta$ & $r^{\prime}$ \\
\hline NNCM & 0.2415 & 0.9077 & 7 \\
NSGA-II: p20g100 & 0.3110 & 0.8193 & 20 \\
NSGA-II: p50g40 & 4.9185 & 1.4724 & 48 \\
NSGA-II: p100g20 & 0.4449 & 1.0788 & 70 \\
\hline
\end{tabular}

One of the advantages of the improved GBNM, which makes it comparable to population-based methods, is that it not only returns one global solution, but also provides a set of local optima called a local set. Nondominated points in the local set can be used to gain more insight into the shape of the Pareto frontier. Figure 8-b shows the local optima found during the optimization of the problem at hand. The local set provides additional non-dominated solutions.

\subsubsection{Analysis of the Neighbourhood of the Optimal Solutions}

Verifying the global optimality of the solutions is a very difficult task; however it is possible to investigate the neighbourhood of the solutions to verify their local optimality. The neighbourhood of a solution is specified by all possible solutions that can be obtained when only one of the design variables is varied. As examples, this is applied to points A, B, C. Figure 9 shows the neighbourhood of the optimal solutions when all design variables are changed one at a time. Apparently, all the solutions found are non-dominated in that neighbourhood.
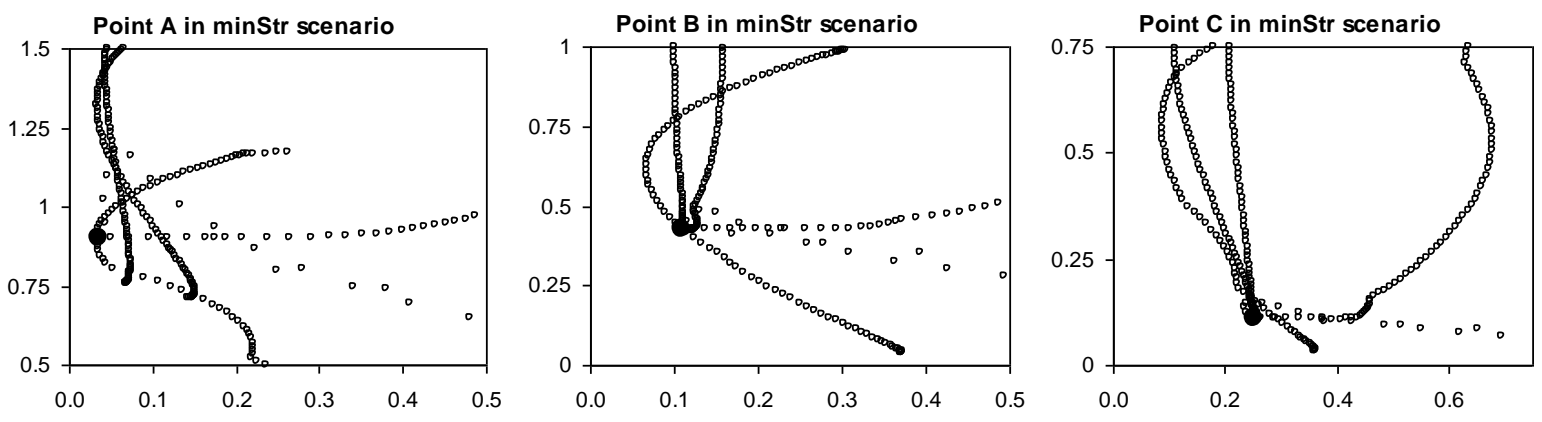

Figure 9. Neighbourhood of points $A, B$, and $C$ in normalized criterion space $\left(F_{S} V s . F_{m}\right)$.

In this section, the design of a composite bracket has been simultaneously optimized for structural and manufacturing objectives. The results were validated by comparing them with an enumeration search and an evolutionary optimization method and also by exploring the neighbourhood of the solutions. The result not only attests to the ability of the algorithm to grab the general form of the Pareto frontier but also shows that the proposed $\mathrm{MOO}$ is able to find better results than those obtained with an evolutionary MOO (i.e. NSGA-II). The trade-off between structural and manufacturing objective also proves the necessity of a multi-objective approach for composite material design.

\subsection{Discussion}

The overall performance of a method is controlled by the parameters defined by the user. This section discusses how user-defined parameters may affect the performance of NNCM and NSGA-II.

If NSGA-II is used with a limit on the total number of function evaluations $(N)$, the only parameter left for the designer to select is the population size $(p)$, or the number of generations $(g)$ considering that $N=p g$. In NSGA-II, the user does not have control over the number of non-dominated solutions, $q$, which is usually known, by experience, to be close to the population size, $q \approx p$ (see Table 2 for 
examples). If only a small number of non-dominated solutions are required, a small population should be utilized. This choice is at the risk of losing the diversity of the population and converging to non-optimal solutions. On the other hand, using a large population size reduces the number of generations, but it may not converge to a promising set of solutions. Similarly, the total number of function evaluations $(N)$ performed in NNCM is

$N=q(2 l r)$,

$q$ : Number of non-dominated solutions desired by the user;

$l$ : Number of N-M iterations for each local optimization process;

$r$ : Number of restarts in CGBNM

If $q$ is small, which is the case we are interested in, there would be enough number of function evaluations for each single objective optimization to find a point close to the Pareto frontier. This explains why in the numerical example solved in this paper NNCM outperforms NSGA-II. If more non-dominated solutions are required, NSGA-II will be a better choice.

Regarding the number of function evaluations, $N$, and number of non-dominated points required, $q$, it is suggested to use NNCM when $q$ and $N$ are small. In contrast, when $N$ and $q$ are large numbers, NSGA-II is recommended. The threshold between small and large values for $N$ and $q$ is problem dependent and must be found by experience. Figure 10 plots these regions. The top left quarter corresponds to the case where a small number of function evaluations is required to obtain a large number of non-dominated solutions. In this region, none of the considered algorithms are able to obtain a good solution, but comparatively NSGAII has been observed to yield better results. In contrast, at the bottom right quarter of the chart, both methods are expected to have a similar performance, although NSGA-II might be selected due to its simplicity.

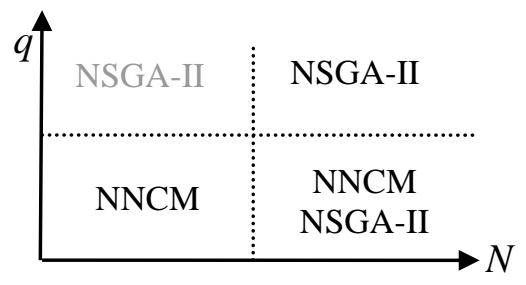

Figure 10. Suggested optimization method regarding the maximum number of function evaluations $(N)$ and number of non-dominated solutions required $(q)$

For a function with a large number of local optima, the local search embedded in NNCM may converge to a non-optimal solution. This drawback amplifies even more when non-linear constraints are imposed by the problem. Figure 8-b shows that, among all the local solutions found by CGBNM, many are located on the boundary of the feasible region (the imaginary lines passing through points A, B, and C in Figure 8-b). To make a more efficient single-objective optimization search by CGBNM, a more efficient constraint handling method is required.

\section{Conclusion}

In order to find a set of Non-dominated solutions for a composite design problem, the Normalized Normal Constraint Method (NNCM) and a local-global optimization method called Constrained Globalized Bounded Nelder-Mead (CGBNM) are coupled in this paper. The proposed method is compared to an evolutionary multi-objective optimization method by solving the simultaneous structural and manufacturing optimization of a Z-shaped composite bracket. This type of problem has two following characteristics: 1) function evaluation is time consuming, thus performing only a small number of function evaluation is possible, 2) the designer does not need a large number of non-dominated solutions. For such problems, the NNCM is found to be more efficient than a population-based method, considering the proximity to the true Pareto frontier and the uniform spread of the solutions. Comparing the results with an enumeration search and investigation of the neighbourhood of the optimal solutions also confirms that the proposed method is able to accurately and efficiently find non-dominated solutions very close to the Pareto frontier. The solutions found by the proposed algorithm clearly dominate the ones obtained by NSGA-II. The corresponding geometries, fiber orientations, and performance criteria of the optimal solutions are 
plotted, showing that the trade-off between structural and manufacturing objectives exist and must be considered during the design process. Having a set of non-dominated solution helps the designer to better understand this trade-off and have some alternative designs before making the final decision.

\section{References}

- Abouhamze M Shakeri M (2007) Multi-objective stacking sequence optimization of laminated cylindrical panels using a genetic algorithm and neural networks, Composite Structures 81(2): 253--263

- Adali S (1983) Multiobjective design of an antisymmetric angle-ply laminate by non-linear programming, ASME Journal of Mechanisms, Transmissions, and Automation in Design 105:214--19

- Adali S, Walker M, Verijenko VE (1996) Multiobjective optimizati.on of laminated plates for maximum prebuckling, buckling and postbuckling strength using continuous and discrete ply angles, Composite Structures 35: 117--130

- Costin DP, Wang BP (1993) Optimum design of a composite structure with manufacturing constrains. Thin-walled Structures 17: 185--202

- Deb K (2001) Multi-objective optimization using evolutionary algorithms, John Wiley \& Sons, LTD, Chichester, New York, Weinheim, Brisbane, Singapore, Toronto.

- Deb K, Pratap A, Agarwal S, Meyarivan T (2002) A fast and elitist multiobjective genetic algorithm: NSGA-II, IEEE Transactions on Evolutionary Computation, 6(2): 182--197

- Deka DJ, Sandeep G, Chakraborty D, Dutta A (2005) Multiobjective optimization of laminated composites using finite element method and genetic algorithm, J of Reinforced Plastics and Composites 24(3):273-285

- Jyotideka D, Sandeep G, Chakraborty D, Dutta A (2005) Multiobjective optimization of laminated composites using finite element method and genetic algorithm. J. Reinforced Plastics and Composites 24(3): 273--285

- Ghiasi H, Pasini D, Lessard L (2008) Constrained globalized Nelder-Mead method for simultaneous structural and manufacturing optimization of a composite bracket, J Composite Materials 42(7): 717--736

- Henderson JL, Gurdal Z, Loos AC (1999) Combined structural and manufacturing optimization of stiffened composite panels. J of Aircraft 36(1): 246--254

- Humphrey DG, Wilson JR (2000) A revised simplex search procedure for stochastic simulation response surface optimization. INFORMS J on Computing 12(4): 272--283

- Kere P, Koski J (2002) Multicriterion optimization of composite laminates for maximum failure margins with an interactive descent algorithm. Struct Multidisc Optim 23: 436--447

- Kere P, Lyly M, Koski J (2003) Using multicriterion optimization for strength design of composite laminates. Composite Struc 62: 329--333

- Kere P, Lento J (2005) Design optimization of laminated composite structures using distributed grid resources, Composite Structures 71(3-4): 435--438

- Lagarias JC, Reeds JA, Wright MH, Wright PE (1998) Convergence properties of the Nelder-Mead simplex method in low dimensions. SIAM J of Optim 9(1): 112--147

- Le Riche R, Saouab A, Breard J (2003) Coupled compression RTM and composite layup optimization. Comp Sc and Tech 63(15): 2277--2287

- Luersen MA, Le Riche R (2004) Globalized Nelder-Mead method for engineering optimization. Computers and Struc 82: 2251--2260

- Manne PM Tsai SW (1998) Design optimization of composite plates: Part II-structural optimization by plydrop targeting, J of Composite Materials 32(6): 572--98

- Marler RT, Arora JS (2004) Survey of multiobjective optimization methods. Struct Multidisc Optim 26: 369--395

- Megson THG (1999) Aircraft structures for engineering students. 3rd Ed, JW Arrowsmith Ltd Bristol, England

- Messac A, Ismail-Yahaya A, Mattson CA (2003) The normalized normal constraint, method for generating the Pareto frontier. Struc Multidisc Optim 25(2) 86--98

- Miettinen K (1999) Nonlinear multiobjective optimization, Kluwer Academic Publishers, Boston.

- Mohan Rao AR, Arvind N (2005) A scatter search algorithm for stacking sequence optimisation of laminate composites. Composite Struc 70: 383--402

- Nelder JA and Mead R (1965) Simplex method for function minimization. Computer J 7(4): 308-313 
- Omkar SN, Mudigere D, Naik GN, Gopalakrishnan S (2008) Vector evaluated particle swarm optimization (VEPSO) for multi-objective design optimization of composite structures, Computers and Structures 86:1--14

- Pagano NJ, Pipes RB (1989) Interlaminar response of composite materials. Composite Mat Series, Vol 5

- Pareto V (1906) Manuale di economica politica, Societa Editrice Libraria, Milan, translated into English by AS Schwier as Manual of Political Economy, edited by AS Schwier and AN 1971. New York: AM Kelley

- Park CH, Lee WI, Kim SJ, Han WS, Vautrin A. (2001) Simultaneous optimization of design and manufacture of composite structures. Collection of Technical Papers AIAA/ASME/ASCE/AHS/ASC Struc, Structural Dynamics and Materials Conference, 1: 186--196

- Park CH, Lee W, Han WS, Vautrin A (2003) Weight minimization of composite laminated plates with multiple constraints. Composites Sc Tech 63(7): 1015--1026

- Park CH, Lee W, Han WS, Vautrin A (2004) Simultaneous optimization of composite structures considering mechanical performance and manufacturing cost. Composite Struc 65(1): 117--127

- Park CH, Lee W, Han WS, Vautrin A (2005) Multiconstraint optimization of composite structures manufactured by resin transfer molding process. J Composite Mat 39(4): 347--374

- Pelletier JL, Vel SS (2006) Multi-objective optimization of fiber reinforced composite laminates for strength, stiffness and minimal mass. Computers and Struc 84: 2065--2080

- Saravanos DA, Chamis CC (1992) Multiobjective shape and material optimization of composite structures including damping, AIAA 30(3): 805--813

- Srinivas N, Deb K (1995) Multi-Objective function optimization using non-dominated sorting genetic algorithms. Evolutionary computation 2(3): 221--248

- Suresh S, Sujit PB, Rao AK (2007) Particle swarm optimization approach for multi-objective composite box-beam design, Composite Structures, 81(4): 598--605

- Tsai SW (1992) Theory of composites design, think composites. a division of ILT corporation, Dayton, Paris, Tokyo

- Walker M, Reiss T, Adali S (1997) Multiobjective design of laminated cylindrical shells for maximum torsional and axial buckling loads, Computers and Structures 62(2): 237--242

- Walker M Smith R (2003) A technique for the multiobjective optimization of laminated composite structures using genetic algorithms and finite element analysis, Composite Structures 62(1): 123--128

- Wang K, Kelly D, Dutton S (2002) Multi-objective optimisation of composite aerospace structures. Composite Struc 57(1): 141--148

- Wang BP, Costin DP (1992) Optimum design of a composite structure with three types of manufacturing constraints. AIAA J 30(6): 1667--1669

- Watkins RI, Morris A (1987) A multi-criteria objective function optimization scheme for laminated composites for use in multilevel structural optimization schemes, Comput Methods Appl Mech Engrg 60: $233--51$

\section{Appendix 1: \\ Modeling and Simulation of the Composite Bracket}

To evaluate the objectives of a composite bracket design problem, an appropriate processing simulation and structural analysis are required. For this purpose, a semi-analytical model is developed in MATLAB ${ }^{\odot}$. The model should be able to evaluate objectives and constraints including the load factor (R), the vertical deflection $(\delta)$, the spring-in $(\Delta \theta)$, and the delamination factor $(D)$.

The load factor is calculated using first-ply-failure of the classical lamination theory and Hashin stressbased failure criterion [Tsai 1992]. Vertical deflection is calculated by numerical integration and energy method [Megson 1999]. The spring-in, which is the angular deformation of a part after demoulding, is a function of cure shrinkage and thermal expansion, and is calculated using the following equation:

$\Delta \theta=\theta\left[\left(\frac{\left(\alpha_{l}-\alpha_{t}\right) \Delta T}{1+\alpha_{t} \Delta T}\right)+\left(\frac{\phi_{l}-\phi_{t}}{1+\phi_{t}}\right)\right]$ 
where $\Delta \theta$ is spring-in, $\theta$ is the angle of the bracket, $\Delta T$ is the temperature difference between the cure temperature and the room temperature. $\phi$ and $\alpha$ are coefficients of shrinkage and thermal expansion, subscripts $l$ and $t$ respectively stand for longitudinal and through thickness directions.

Delamination is a critical mode of failure in composite materials, and it is due to the interlaminar stresses between subsequent laminates. In a flat plate, interlaminar stresses are created only by the free-edge effect [Pagano and Pipes 1989], but in a curved part, the 3D stress field also creates significant interlaminar stresses that may cause delamination at the curved regions. In this paper, we adopt a convenient, albeit crude, model of free edge interlaminar stresses by Pagano and Pipes (1989). In this model the interlaminar normal stress is estimated as shown in Figure A-2.

Interlaminar normal stresses created by the angle-shape effect are shown in Figure A-3. Sequentially solving the equilibrium equations for all layers, starting form the innermost layer results in an interlaminar normal stress between layers $n$ and $n+1$ :

$$
\sigma_{z, n-(n+1)}=\sum_{k=1}^{n} \sigma_{x, k} t_{k} / R+\sum_{k=1}^{n} t_{k}
$$

where, $t_{i}$ shows the thickness of $\mathrm{i}^{\text {th }}$ layer, and $R$ is the inner radius of the curved part. Interlaminar shear stresses are of minor importance with respect to interlaminar normal stresses [Tsai 1992], thus an approximation of shear stress in a prismatic member under a transverse load is used.

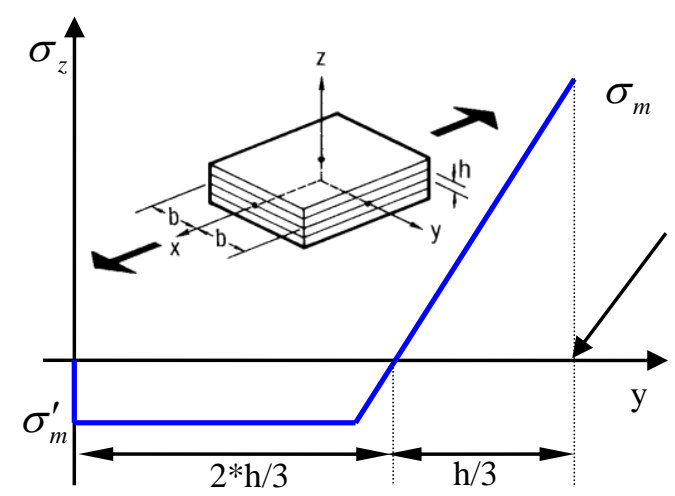

$$
\begin{aligned}
& \sigma_{m}^{\prime}=\frac{1}{5} \sigma_{m}, \\
& \sigma_{m}=\sigma_{m}(z)=45 M(z) / 14 h^{2}, \\
& M(z)=\int_{z}^{h} \sigma_{y}(\xi)(\xi-z) d \xi, \\
& \sigma_{y}(z)=\text { Transverse stress }
\end{aligned}
$$

Figure A-2. Approximate distribution of interlaminar normal stress at a free edge
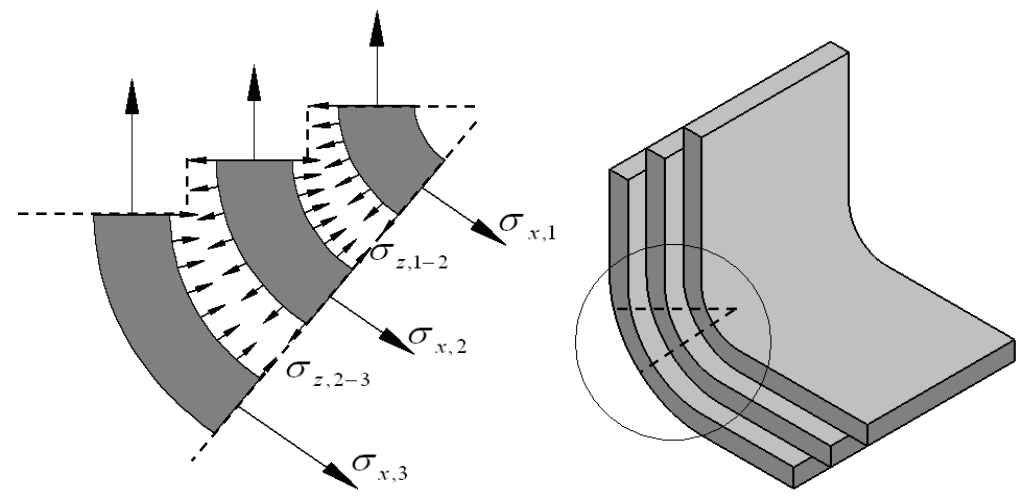

Figure A-3. Interlaminar normal stresses in the curved region of an angle-shaped flange 